\title{
Exploring new avenues in high repetition rate table-top coherent extreme ultraviolet sources
}

\author{
Steffen Hädrich $^{1,2}$, Manuel Krebs ${ }^{1}$, Armin Hoffmann ${ }^{1}$, Arno Klenke ${ }^{1,2}$, Jan Rothhardt ${ }^{1,2}$, Jens Limpert ${ }^{1,2}$ \\ and Andreas Tünnermann ${ }^{1,2,3}$
}

The process of high harmonic generation (HHG) enables the development of table-top sources of coherent extreme ultraviolet (XUV) light. Although these are now matured sources, they still mostly rely on bulk laser technology that limits the attainable repetition rate to the low kilohertz regime. Moreover, many of the emerging applications of such light sources (e.g., photoelectron spectroscopy and microscopy, coherent diffractive imaging, or frequency metrology in the XUV spectral region) require an increase in the repetition rate. Ideally, these sources are operated with a multi-MHz repetition rate and deliver a high photon flux simultaneously. So far, this regime has been solely addressed using passive enhancement cavities together with low energy and high repetition rate lasers. Here, a novel route with significantly reduced complexity (omitting the requirement of an external actively stabilized resonator) is demonstrated that achieves the previously mentioned demanding parameters. A krypton-filled Kagome photonic crystal fiber is used for efficient nonlinear compression of $9 \mu \mathrm{J}, 250$ fs pulses leading to $\sim 7 \mu \mathrm{J}, 31$ fs pulses at $10.7 \mathrm{MHz}$ repetition rate. The compressed pulses are used for HHG in a gas jet. Particular attention is devoted to achieving phase-matched (transiently) generation yielding $>10^{13}$ photons s$^{-1}(>50 \mu \mathrm{W})$ at $27.7 \mathrm{eV}$. This new spatially coherent XUV source improved the photon flux by four orders of magnitude for direct multi-MHZ experiments, thus demonstrating the considerable potential of this source.

Light: Science \& Applications (2015) 4, e320; doi:10.1038/Isa.2015.93; published online 28 August 2015

Keywords: coherent extreme ultraviolet sources; high average power; high harmonic generation; nonlinear compression; ultrafast laser

\section{INTRODUCTION}

High harmonic generation (HHG) offers an elegant approach to achieve table-top sources of coherent extreme ultraviolet (XUV) or even soft X-ray radiation ${ }^{1}$. Although this process has been known for almost 30 years, it is still a subject of intense research because of the ever-increasing number of possible applications. Moreover, these sources are an alternative to large-scale facilities, such as free electron lasers or synchrotrons, which provide limited user access. All of these sources are used in diverse fields, e.g., in physics, biology, chemistry, material science, and others. This application-oriented approach has defined two major challenges in the field of HHG, which involved either increasing the energy per XUV pulse or the repetition rate $^{2,3}$. The latter challenge is particularly important for photoelectron spectroscopy and microscopy in which space-charge effects need to be avoided ${ }^{3,4}$. Other possible applications are coherent diffractive imaging ${ }^{5,6}$, coincidence experiments ${ }^{7}$, or frequency metrology, in which multi-10 MHz lasers with a stabilized frequency comb are used ${ }^{8}$. Generally, there is a great demand for compact, cost-effective and reliable sources that can also be used by scientists who are not laser experts, which would severely broaden their applications.

HHG relies on the interaction of a high-intensity $\left(10^{13}-10^{15} \mathrm{~W} \mathrm{~cm}^{-2}\right)$ ultrashort laser pulse with noble gases. The efficiency of the HHG process critically depends on the possibility of achieving phase-matching, i.e., matching the phase velocity of the driving laser field and the generated XUV field ${ }^{9}$. The phase-mismatch is determined by the dispersion of the neutral atoms and free electrons, the intrinsic phase due to the propagation of the free electron wave-packet as well as a geometrical term, which is caused by the Gouy phase-shift ${ }^{10,11}$. The dispersion terms (neutral atoms, free electrons) have opposite signs and are density dependent. Therefore, phase-matching can be transiently achieved (i.e., for a certain instance of time of the laser pulse) by adjusting the pressure of the generation gas to balance the terms. However, this approach only works when the ionization of the gas medium is maintained below a so-called critical ionization, which is a few percent depending on the gas species and harmonic order ${ }^{1,9}$. This critical ionization level restricts the intensity that can be used for HHG to a maximum value that significantly increases with decreasing pulse duration. Consequently, ultrashort pulses ( $<10$ cycles) will allow the use of a higher intensity, which in turn increases the single atom response while still achieving phase-matching and high efficiencies $\left(>10^{-6}\right)^{1}$. Therefore, the Ti:Sapphire-based laser systems, due to their ultrashort pulse duration, are still considered the work horse in this field ${ }^{1}$.

However, due to the thermo-optical constraints, the repetition rate does not exceed a few kilohertz (a few Watt of average power).

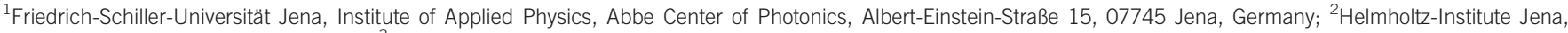
Fröbelstieg 3, 07743 Jena, Germany and ${ }^{3}$ Fraunhofer Institute for Applied Optics and Precision Engineering, Albert-Einstein-Straße 7, 07745 Jena, Germany 
However, novel laser concepts, such as thin-disk ${ }^{12,13}$, innoslab ${ }^{14}$, and fiber lasers ${ }^{15}$, have shown impressive advances in their performance and reached the kilowatt level of average power during pulsed operation. All of these architectures are based on ytterbium-doped laser materials that limit the attainable pulse duration to a few hundred femtoseconds. Nevertheless, early experiments on HHG with fiber lasers have already indicated the great potential of these sources ${ }^{16,17}$, which has also been validated by the recently measured photon flux of $4.5 \times 10^{12}$ photons $\mathrm{s}^{-1}$ over several harmonics at $100 \mathrm{kHz}$ repetition rate $^{18}$. However, as noted above, the rather long pulse duration hinders efficient HHG, suggesting the need for post-compression techniques, such as nonlinear compression in noble gas-filled glass capillaries ${ }^{19}$. Nonlinear compression has already shown its capability to be operated with high average powers ${ }^{20}$. Additionally, the use of fiber-chirped pulse amplifiers in combination with nonlinear compression progressively increases the photon flux obtained via HHG. More than $3 \times 10^{13}$ photons $\mathrm{s}^{-1}$ per harmonic $(140 \mu \mathrm{W})$ at $30 \mathrm{eV}^{21}$ and more than $3 \times 10^{9}$ photons $\mathrm{s}^{-1}$ within a $1 \%$ bandwidth at $120 \mathrm{eV}^{22}$ have been generated recently, which can now be considered the highest photon flux HHG sources in this spectral range. The repetition rate in those experiments was $600 \mathrm{kHz}$ and $100 \mathrm{kHz}$, respectively, but a further increase to the multi- $\mathrm{MHz}$ level is required to address the previously mentioned applications.

To meet this requirement, passive external resonators that enhance the incoming power by a factor of up to 1000 have been proposed. Therefore, a low energetic laser system can be employed at repetition rates ranging from $10 \mathrm{MHz}^{23}$ to $154 \mathrm{MHz}^{8}$. However, they require an active stabilization of the resonator to maintain the enhancement. Moreover, the harmonics have to be generated directly in the resonator, which necessitates an out-coupling mechanism for the harmonics, adding additional experimental complexity ${ }^{8,24}$. The occurring ionization of the generation gas puts further constraints on the achievable enhancement and phase-matching conditions that challenges further development ${ }^{24,25}$. A second approach relies on plasmonic field enhancement that potentially allows operation directly with laser oscillators $^{26}$. Here, a specially designed nanostructure leads to field enhancement near the edges of these structures that can provide the required intensity for HHG. However, the usability of the latter is heavily debated in the community, and it remains unclear whether this approach is feasible ${ }^{27}$. A third possibility is to directly use high repetition rate laser systems. This requires tighter focusing to achieve the intensities necessary for HHG, which also severely increases the contribution of the geometrical phase-mismatch term (see above). In combination with the reduced focal spot size and generation volume, it has been suggested that the efficiency will be significantly lower than in loose focusing geometries ${ }^{28}$. Indeed, the lack of phase-matching explains the poor efficiency of previous multi- $\mathrm{MHz}$ repetition rate

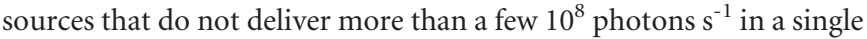
harmonic ${ }^{28,29}$. However, investigations of the phase-matching behavior suggest that this can be overcome by properly choosing the experimental conditions, most notably a high-density gas target, to achieve the same pressure-length product as in loose focusing geometries ${ }^{10,11}$. Recent experiments have successfully demonstrated efficient conversion $\left(8 \times 10^{-6}\right)$ in a tight focusing geometry ${ }^{11}$.

In this article, nonlinear compression of $9 \mu \mathrm{J}, 250 \mathrm{fs}$ pulses in a krypton-filled Kagome photonic crystal fiber (PCF) is demonstrated. After spectral broadening in the PCF, $\sim 7 \mu \mathrm{J}, 31 \mathrm{fs}$ pulses are achieved at up to $10.7 \mathrm{MHz}$ repetition rate. This laser system is used to demonstrate transiently phase-matched HHG. More than $10^{13}$ photons s ${ }^{-1}$ $(>50 \mu \mathrm{W})$ are achieved in a single harmonic at $27.7 \mathrm{eV}$, which is an improvement of four orders of magnitude for a directly driven multi$\mathrm{MHz}$ repetition rate $\mathrm{HHG}^{28,29}$, and it provides a photon flux that is higher than that delivered by standard $\mathrm{kHz}$ laser systems ${ }^{30}$ and intracavity HHG within that wavelength range $e^{8,24,31}$. The use of a lowenergy driving laser with a compact nonlinear compression setup not only has the potential to significantly reduce the complexity of high repetition rate HHG systems but will also enable efficient HHG at $10 \mathrm{MHz}$ repetition rate, a regime so far only addressable with enhancement cavities.

\section{MATERIALS AND METHODS}

The experimental setup of the nonlinear compression stage followed by a HHG experiment is shown in Figure 1. The front end is a fiber chirped pulse amplification system that optionally allows for the coherent combination of up to four main amplifier channels (CCFCPA $)^{32}$. This system is chosen because of its short pulse duration of $250 \mathrm{fs}$, which also enables shorter pulses after the nonlinear compression.

It is important to emphasize that only $8-9 \mu \mathrm{J}$ of pulse energy is used at up to $10.7 \mathrm{MHz}$ repetition rate ( $90 \mathrm{~W}$ average power). Therefore, this system, which was used due to its availability, can be easily replaced by compact, turn-key fiber laser systems ${ }^{33,34}$ or with stateof-the-art thin-disk oscillators ${ }^{35,36}$. The nonlinear compression stage is necessary to achieve the required ultrashort pulse durations for efficient $\mathrm{HHG}^{1,9}$. The pulses from the CC-FCPA are directed through a telescope and a focusing lens and are then coupled into the Kagome fiber (Figure 1). This new type of PCF offers a relatively large core (30-60 $\mu \mathrm{m})$, low propagation loss and allows studying a variety of nonlinear effects ${ }^{37}$. Interestingly, the Kagome PCF has been recognized as the perfect waveguide for nonlinear compression of $\mu \mathrm{J}$-level pulses $^{38,39}$, whereas conventional capillaries have extremely high propagation losses due to the required small diameter. Furthermore, they can be operated at high average powers ${ }^{40}$. Here, a commercially available Kagome (PMC-C-Yb-7C, GLOphotonics Limoge, France) with a mode-field diameter of $40 \mu \mathrm{m}$ and a length of $1 \mathrm{~m}$ is used. The Kagome

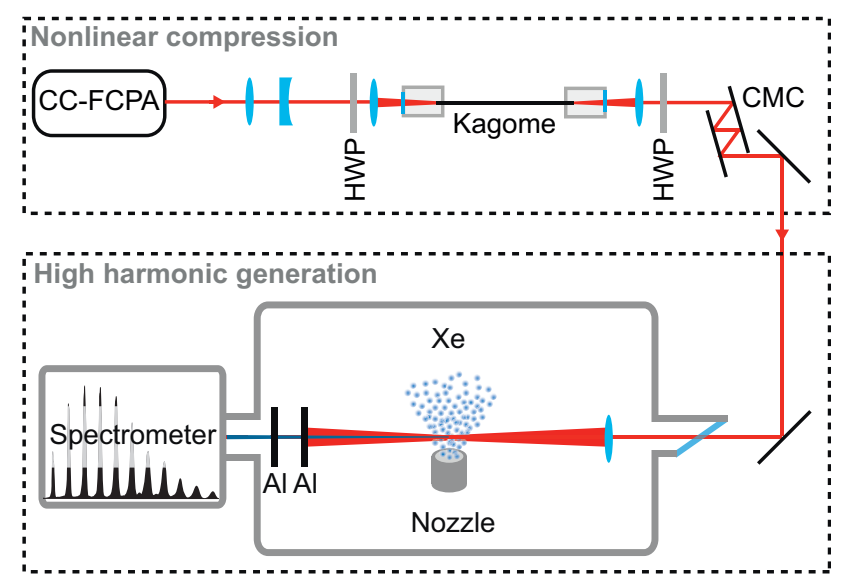

Figure 1 The pulses from a fiber chirped pulse amplifier (CC-FCPA) are coupled into a $1 \mathrm{~m}$ long Kagome PCF, which is filled with krypton gas. After spectral broadening, the pulses are compressed by a chirped mirror compressor and then directed to a vacuum chamber for HHG. A lens focuses the pulses to an intensity of $7.4 \times 10^{13} \mathrm{~W} \mathrm{~cm}^{-2}$ (11 $\mu \mathrm{m}$ focal spot radius) in a xenon gas jet ejected from a nozzle, which is mounted on an $x-y-z$ translation stage. Two $200 \mathrm{~nm}$ thick aluminum filters separate the generated harmonics and the remaining infrared light. The spatial-spectral analysis of the harmonics is performed with a flat-field grating spectrometer (HWP - half wave plate). 


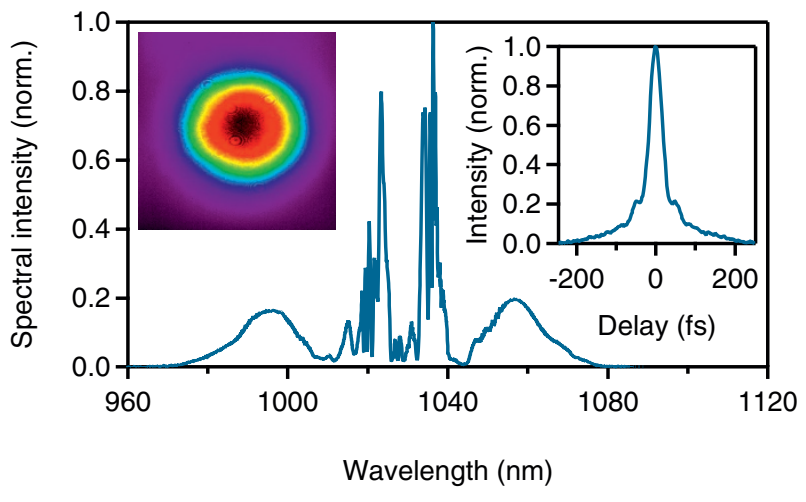

Figure 2 The broadened spectrum at a repetition rate of $10.7 \mathrm{MHz}$ (Left inset: beam profile of the collimated beam. Right inset: autocorrelation of the compressed pulse)

fiber is mounted on a water-cooled V-groove to ensure stable high average power operation. Before coupling into the fiber, a first halfwave plate (HWP) adjusts the polarization so that linear polarization is obtained behind the fiber. Subsequently, another HWP rotates to ppolarization, which is required for the chirped mirror compressor (Figure 1). Typically, approximately $3 \%$ of the emitted power is found to be in the incorrect polarization direction when operating the setup as described here. This excellent linear polarization, in addition to ultrashort pulses, is a crucial prerequisite for achieving efficient $\mathrm{HHG}^{41}$.

The average power transmission through the setup is greater than $80 \%$ including coupling, propagation, and the mirror compressor. To achieve spectral broadening, the fiber is statically filled with 7 bar of krypton gas. When coupling the $\sim 9 \mu \mathrm{J}$ pulses into the fiber, self-phase modulation in the krypton gas produced a rms-bandwidth of approximately $49 \mathrm{~nm}$ after the fiber (Figure 2). Further spectral broadening was not possible with this specific fiber due to the occurrence of other nonlinear effects. However, it has already been demonstrated that proper fiber designs allow for the achievement of $10 \mathrm{fs}$ pulses ${ }^{39}$. Temporal compression is then achieved using four reflections on highly dispersive and broadband ( $-350 \mathrm{fs}^{2}$ per reflection, 980-1080 $\mathrm{nm}$ ) chirped mirrors. The compressed pulse is characterized by an autocorrelation width of $\sim 43 \mathrm{fs}$ (inset in Figure 2) that corresponds to a pulse duration of $\sim 31$ fs (transform-limit: $30 \mathrm{fs}$ ). The energy of the compressed pulse is $7.1 \mu \mathrm{J}(76 \mathrm{~W}$ average power at $10.7 \mathrm{MHz}$ ). Approximately $70 \%$ of the total energy (including pre-/post-pulses of the CC-FCPA) is contained in the main pulse, leading to a peak power of approximately $140 \mathrm{MW}$. Due to the use of a waveguide for spectral broadening, the spatial quality of the output beam is excellent as indicated by the collimated beam profile after the chirped mirror compressor (inset in Figure 2).

After this nonlinear compression stage, the pulses are steered into a vacuum chamber for HHG (Figure 1). A Brewster-angled entrance window is used to eliminate reflection losses. The beam is focused to a radius $\left(1 / \mathrm{e}^{2}\right.$-intensity) of $\sim 11 \mu \mathrm{m}$, leading to an estimated peak intensity of $7.4 \times 10^{13} \mathrm{~W} \mathrm{~cm}^{-2}$. The noble gas for HHG is injected by a $65 \mu \mathrm{m}$ nozzle continuously backed with xenon at several bar of pressure. The choice of the gas jet size is explained in more detail in the following section (Results and Discussion). After the HHG, the fundamental laser beam and the XUV light co-propagate until a pair of $200 \mathrm{~nm}$ thick aluminum filters reflect the laser light and partly transmit the harmonics, which are then analyzed with a flat-field grating spectrometer to observe the spatial and spectral characteristics.

The average power of the harmonics is obtained by spatially and spectrally integrating over a single harmonic and accounting for the transmission characteristics of the detection system as described in Ref. 21. This includes the diffraction efficiency of the spectrometer grating, the characteristics of the detector (CCD, characterized by the manufacturer) and the measured filter transmission (see Supplementary material).

\section{RESULTS AND DISCUSSION}

The optimization of the experimental conditions is performed at $234 \mathrm{kHz}$ repetition rate. To achieve phase-matching in a tight focusing geometry, the scaling considerations that are described in the Ref. 10,11 are implemented. Basically, they relate important quantities, such as the HHG medium length and gas density, for different focusing geometries with the assumption that the same peak intensity, pulse duration, and central wavelength are used. Following these guidelines and using the recently demonstrated efficient HHG of post-compressed fiber lasers as a basis ${ }^{21}$, a $65 \mu \mathrm{m}$ nozzle size and xenon gas is chosen. Experimentally, the position of the gas nozzle relative to the focus and the backing pressure are optimized for maximum signal. This results in the jet being positioned as close as possible to the beam and slightly behind the focus, which guarantees that the short trajectories are phase-matched and that well-defined spatial profiles with low divergence are obtained ${ }^{42}$. For a better understanding of the phasematching conditions, the signal of the harmonics is studied with respect to the backing pressure, e.g., for the two strongest harmonics $\mathrm{H} 21$ and $\mathrm{H} 23$ (Figure 3). As shown in Figure 3, the maximum signal is obtained at 1-1.2 bar, which is also in good agreement with the predictions of the scaling laws ${ }^{10,11}$. At these experimental conditions,

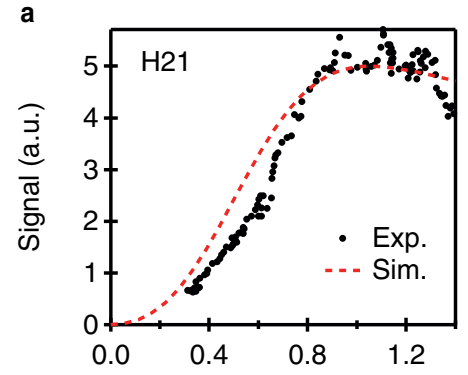

Pressure in interaction region (bar) b

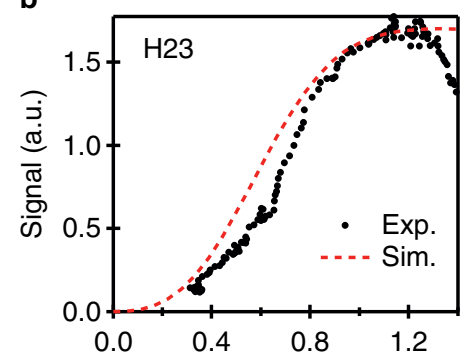

Pressure in interaction region (bar)

Figure 3 The signal of the 21st (a) and 23rd (b) harmonic is recorded for varying pressures (black dots). The signal build-up is compared with a phase-matching model (dashed red curves), which is described in the text. 


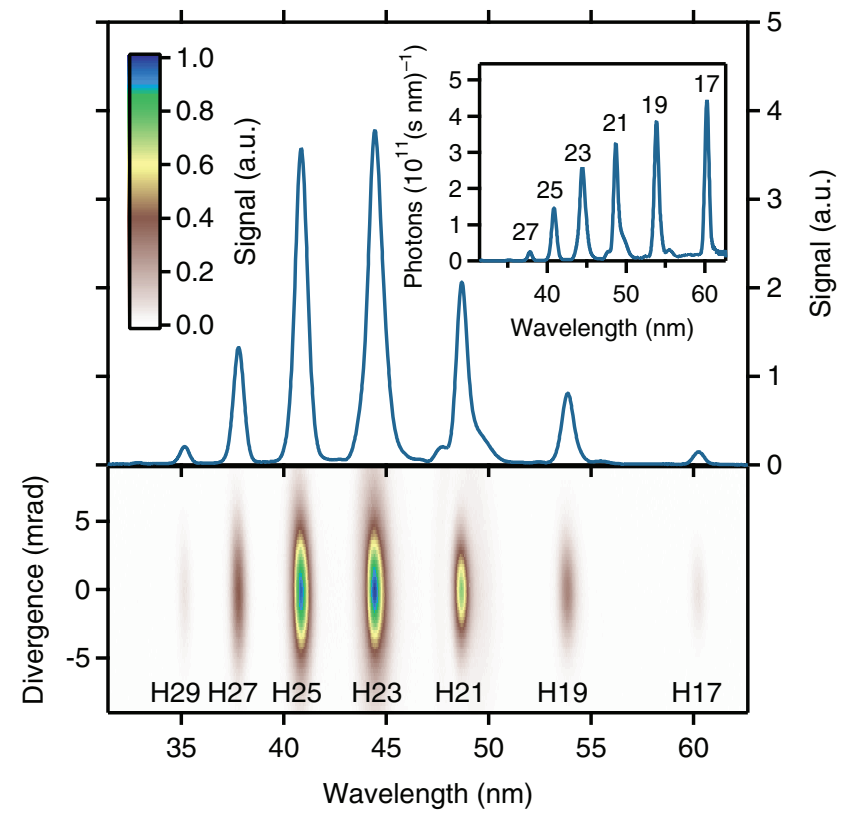

Figure 4 The spatially ( $x$-axis) and spectrally (y-axis) resolved high harmonics generated at $234 \mathrm{kHz}$ (lower panel). The experimental conditions are described in the text. The upper panel shows the spatial integration of the lower panel (inset: calibrated spectrum).

well-defined harmonics with a Gaussian beam profile are generated (Figure 4) as expected for phase-matched generation. The phasematching conditions can be further investigated using a one-dimensional model that was introduced by Constant et al. and Kazamias et al. ${ }^{43,44}$ This model calculates the signal of the $q$ th harmonic on-axis and includes the pressure- and time-dependent wave-vector mismatch, $\Delta k(t, p)=q \times k_{0}-k_{q}$, absorption effects and an empirical model of the single atom response ${ }^{44}$. The calculation of the wavevector mismatch is performed at the focus position where the contribution of the dipole term, $\left(\Delta k_{\text {dipole }}=-\alpha \times \partial I(z) / \partial z\right)$, vanishes.
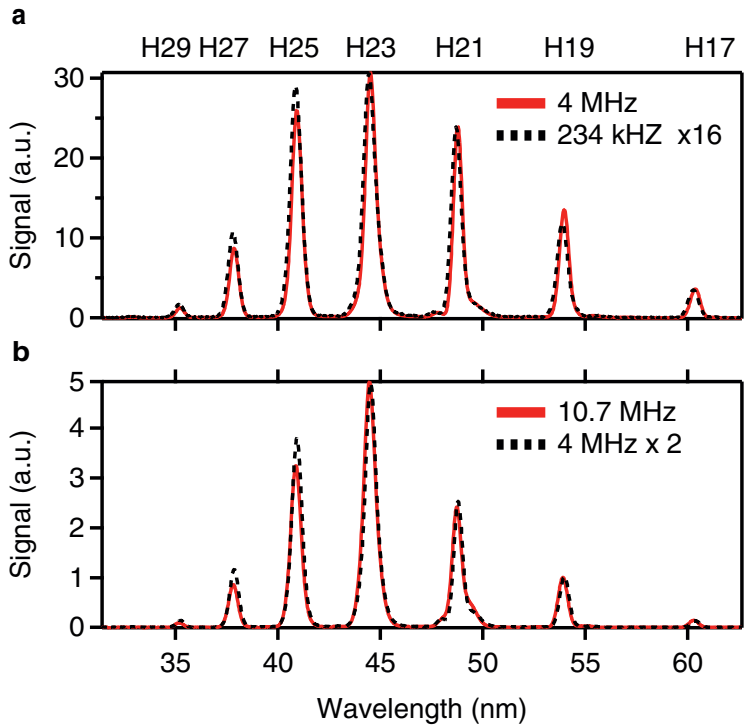

Figure 5 The signal of the high harmonics is compared for repetition rates of 234 $\mathrm{kHz}$ and $4 \mathrm{MHz}$ (a) and $4 \mathrm{MHz}$ and $10.7 \mathrm{MHz}$ (b).
Table 1. HHG characteristics at a $10.7 \mathrm{MHz}$ repetition rate

\begin{tabular}{lccccc}
\hline Harmonic order & $\mathrm{H} 17$ & $\mathrm{H} 19$ & $\mathrm{H} 21$ & $\mathrm{H} 23$ & $\mathrm{H} 25$ \\
\hline Wavelength $(\mathrm{nm})$ & 60 & 54 & 49 & 45 & 41 \\
Average power $(\mu \mathrm{W})$ & 27.6 & 34.8 & 39.4 & 51.1 & 25.5 \\
Photons s $^{-1}\left(10^{12}\right)$ & 8.3 & 9.4 & 9.7 & 11.4 & 5.3 \\
${\text { Conversion efficiency }\left(10^{-7}\right)}^{3}$ & 3.6 & 4.6 & 5.2 & 6.7 & 3.4 \\
\hline
\end{tabular}

Further details on the calculation of the remaining terms are described elsewhere ${ }^{9-11}$. For the calculation a focal spot size radius of $11 \mu \mathrm{m}$ (1/ $\mathrm{e}^{2}$-intensity), an intensity of $7.4 \times 10^{13} \mathrm{~W} \mathrm{~cm}^{-2}$ and a Gaussian temporal envelope with a full width at half maximum duration of $30 \mathrm{fs}$ are used. Additionally, we consider the residual absorption of the harmonics signal on its way to the detector as described in the supplementary information of the Ref. 21. The required ionization rates are calculated with the established model of Ammosov-DeloneKrainov $(\mathrm{ADK})^{45}$. This calculation reveals that the intensity used leads to a fraction of ionized medium that is larger than the so-called critical ionization ${ }^{9}$. Therefore, the phase-matching condition is transiently fulfilled at the leading edge of the ultrashort laser pulse, where the coherence length, $\left(L_{\mathrm{coh}}=\pi / \Delta k\right)$, is longer than the medium length. The calculated harmonics signal (red dashed curves in Figure 3 ) shows good agreement with the measured signal with respect to the pressure in the interaction region. The pressure in the interaction region for the experimental data is obtained by scaling the recorded backing pressure using a gas-expansion model ${ }^{11,46}$. When phase-matching is achieved, the build-up of the harmonics is only limited by the linear absorption of the generation medium itself. Constant et al. have defined a criterion, i.e., $L_{\text {med }}>3 L_{\text {abs }}$ and $L_{\text {coh }}>5 L_{\text {abs }}$, under which the obtained harmonics signal is more than $50 \%$ of this asymptotic value ${ }^{43}$. The absorption lengths of the harmonics are $L_{\mathrm{abs}}=15 \mu \mathrm{m}$ (H17), $L_{\mathrm{abs}}=$ $20 \mu \mathrm{m}(\mathrm{H} 19), L_{\mathrm{abs}}=26 \mu \mathrm{m}(\mathrm{H} 21)$, and $L_{\mathrm{abs}}=30 \mu \mathrm{m}(\mathrm{H} 23)$ at the optimal experimental conditions ${ }^{47}$, i.e., the maximum obtained signal. This means that the 17th and 19th harmonics are already absorption limited in their build-up. The upper panel of Figure 4 shows the obtained raw spectrum (spatially integrated) that was acquired by the CCD under the optimized conditions. When performing the calibration, the spectral shape changes (see inset of Figure 4) due to the aluminum filter transmission (see Supplementary Information). The calculation of the average power of the respective harmonics reveals that even at the low repetition rate of $234 \mathrm{kHz}$ more than 1.5 $\mu \mathrm{W}\left(>3 \times 10^{11}\right.$ photons $\mathrm{s}^{-1}$, inset of Figure 4$)$ are generated in the strongest generated harmonic $(\mathrm{H} 23,27.7 \mathrm{eV})$.

After the HHG optimization, the scaling behavior with respect to the repetition rate was investigated (Figure 5). For this measurement, the two $200 \mathrm{~nm}$ thick aluminum filters (10 $\mathrm{mm}$ round aperture) are replaced by a $400 \mathrm{~nm}$ thick aluminum filter, which is mounted on a slit and can withstand a very high average power. First, the reference signal is recorded at $234 \mathrm{kHz}$ and then compared with the signal obtained at $4 \mathrm{MHz}$, which is measured to be 16 times stronger (Figure $5 \mathrm{a}$ ). Before further increasing the repetition rate, an additional $200 \mathrm{~nm}$ thick aluminum filter is inserted in the spectrometer chamber, which explains the change in the HHG spectrum in Figure 5b because aluminum absorbs stronger for the low harmonics (see Supplementary Figure S1). A new reference signal at $4 \mathrm{MHz}$ is measured and compared with the signal obtained at the highest repetition rate of $10.7 \mathrm{MHz}$, showing another increase by a factor of 2 (Figure $5 \mathrm{~b}$ ). Using the overall obtained scaling factor of 32 and the calibrated measurement at $234 \mathrm{kHz}$ (inset of Figure 4), the average power and conversion efficiency at $10.7 \mathrm{MHz}$ can be determined. Table 1 lists these values for the 17 th to the 25 th harmonic. Remarkably, more than $50 \mu \mathrm{W}$ of average 
power $\left(>10^{13}\right.$ photons $\left.\mathrm{s}^{-1}\right)$ is obtained in the strongest harmonic $(\mathrm{H} 23,27.7 \mathrm{eV})$, and all of these harmonics possess more than 20 $\mu \mathrm{W}\left(>5 \times 10^{12}\right.$ photons $\left.\mathrm{s}^{-1}\right)$. The achieved conversion efficiency of up to $6.7 \times 10^{-7}(\mathrm{H} 23)$ is close to the typical values $\left(10^{-6}\right)$ achievable with other laser systems ${ }^{44}$ and should be improvable by at least a factor of 2 by further optimizing the experimental conditions. The achieved photon flux is four orders of magnitude larger than that reported in studies on multi- $\mathrm{MHz}$ repetition rate $\mathrm{HHG}$ directly driven with a laser, i.e., without the additional enhancement cavity ${ }^{28,29}$. Moreover, the flux obtained in $\mathrm{H} 23$ is higher than what the state-of-the art enhancement cavities can deliver ${ }^{8,24,31}$ and also exceeds the currently used $\mathrm{kHz}$ systems ${ }^{30}$.

\section{CONCLUSION}

In summary, a promising novel source of high repetition rate coherent XUV radiation has been presented. Nonlinear compression in a Kagome PCF is utilized for achieving $7 \mu \mathrm{J}, 31 \mathrm{fs}$ pulses at up to 10.7 MHz repetition rate. Furthermore, these pulses are used for efficient HHG in a $65 \mu \mathrm{m}$ xenon gas jet. This results in a conversion efficiency of $6.7 \times 10^{-7}$, enabling more than $50 \mu \mathrm{W}$ in a single harmonic $\left(>10^{13}\right.$ photons $\left.\mathrm{s}^{-1}\right)$, which is an improvement of four orders of magnitude compared with the reported multi-MHz systems of a single-pass $\mathrm{HHG}^{28,29}$. The photon flux is not only higher than what is supplied by current Ti:Sapphire technology ${ }^{30}$ but also higher than intra-cavity HHG in the same wavelength range ${ }^{8,24,31}$. Additionally, the demonstrated repetition rate has previously been only accessible with enhancement cavities ${ }^{23}$. The achievement of efficient HHG with low-energy and high repetition rate laser systems has profound implications for a diverse field of HHG applications, e.g., in (multi)dimensional surface science ${ }^{48}$, coincidence detection ${ }^{7}$, or coherent diffractive imaging ${ }^{6,49}$. Moreover, this experimental demonstration paves the way for making HHG sources more compact, cost-effective, reliable, and accessible to non-laser experts (e.g., by replacing our front-end (CC-FCPA) with a compact turn-key fiber laser system ${ }^{33,34}$ or a thin-disk oscillator $\left.{ }^{35,36}\right)$. Future work will aim to combine stateof-the-art kilowatt class femtosecond lasers ${ }^{15}$ with the presented compression scheme to achieve milliwatt-level harmonics. The realization of phase-matched HHG with an even tighter focus and the use of a carrier envelope phase and repetition rate stabilized driver will provide a source with a high photon flux $(\sim 100 \mu \mathrm{W}$ per harmonic) with repetition rates of $40 \mathrm{MHz}$ or more that could then be used for frequency metrology in the XUV.

\section{ACKNOWLEDGEMENTS}

This work was partly supported by the German Federal Ministry of Education and Research (BMBF) and the European Research Council under the European Union's Seventh Framework Programme (FP7/2007-2013)/ERC Grant Agreement No. 240460. Arno Klenke and Jan Rothhardt acknowledge financial support by the Helmholtz-Institute Jena.

1 Popmintchev T, Chen M-C, Arpin P, Murnane MM, Kapteyn HC. The attosecond nonlinear optics of bright coherent X-ray generation. Nat Phot 2010; 4: 822-832.

2 Sansone G, Poletto L, Nisoli M. High-energy attosecond light sources. Nat Phot 2011; 5: 655-663.

3 Südmeyer T, Marchese SV, Hashimoto S, Baer CRE, Gingras G et al. Femtosecond laser oscillators for high-field science. Nat Phot 2008; 2: 599-604.

4 Mathias S, Bauer M, Aeschlimann M, Miaja-Avila L, Kapteyn HC et al. Time-resolved photoelectron spectroscopy at surfaces using femtosecond XUV pulses. In: Bovensiepen U, Petek H, Wolf M, editors. Dynamics at Solid State Surface and Interfaces Vol.1: Current Developments. Wiley-VCH Verlag GmbH \& Co. KGaA; 2010. pp499-535.
5 Miao J, Sandberg R, Song C. Coherent X-ray diffraction imaging. IEEE J Sel Top Quant Electron 2012; 18: 399-410.

6 Zürch M, Rothhardt J, Hädrich S, Demmler S, Krebs M et al. Real-time and Subwavelength Ultrafast Coherent Diffraction Imaging in the Extreme Ultraviolet. Sci Rep 2014; 4: 7356.

7 Zhou X, Ranitovic P, Hogle CW, Eland JHD, Kapteyn HC et al. Probing and controlling non-Born-Oppenheimer dynamics in highly excited molecular ions. Nat Phys 2012; 8: 232-237.

8 Cingöz A, Yost DC, Allison TK, Ruehl A, Fermann ME et al. Direct frequency comb spectroscopy in the extreme ultraviolet. Nature 2012; 482: 68-71.

9 Paul A, Gibson EA, Zhang X, Lytle A, Popmintchev T et al. Phase-matching techniques for coherent soft X-ray generation. Quantum Electron IEEE J 2006; 42: 14-26.

10 Heyl CM, Güdde J, L'Huillier A, Höfer U. High-order harmonic generation with $\mu \mathrm{J}$ laser pulses at high repetition rates. J Phys B At Mol Opt Phys 2012; 45: 074020.

11 Rothhardt J, Krebs M, Hädrich S, Demmler S, Limpert J et al. Absorption-limited and phase-matched high harmonic generation in the tight focusing regime. New J Phys 2014; 16: 033022.

12 Schulz M, Riedel R, Willner A, Düsterer S, Prandolini MJ et al. Pulsed operation of a high average power Yb:YAG thin-disk multipass amplifier. Opt Express 2012; 20: 5038-5043.

13 Saraceno CJ, Emaury F, Heckl OH, Baer CRE, Hoffmann M et al. 275 W average output power from a femtosecond thin disk oscillator operated in a vacuum environment. Opt Express 2012; 20: 23535-23541.

14 Russbueldt P, Mans T, Weitenberg J, Hoffmann HD, Poprawe R. Compact diode-pumped 1.1 kW Yb:YAG Innoslab femtosecond amplifier. Opt Lett 2010; 35: 4169-4171.

15 Eidam T, Hanf S, Seise E, Andersen TV, Gabler T et al. Femtosecond fiber CPA system emitting $830 \mathrm{~W}$ average output power. Opt Lett 2010; 35: 94-96.

16 Boullet J, Zaouter Y, Limpert J, Petit S, Mairesse Y et al. High-order harmonic generation at a megahertz-level repetition rate directly driven by an ytterbiumdoped-fiber chirped-pulse amplification system. Opt Lett 2009; 34: 1489-1491.

17 Hädrich S, Krebs M, Rothhardt J, Carstens H, Demmler S et al. Generation of $\mu$ W level plateau harmonics at high repetition rate. Opt Express 2011; 19: 19374-19383.

18 Cabasse A, Machinet G, Dubrouil A, Cormier E, Constant E. Optimization and phase matching of harmonic generation at high repetition rate. Opt Lett 2012; 37: 46184620.

19 Nisoli M, De Silvestri S, Svelto O. Generation of high energy 10 fs pulses by a new pulse compression technique. Appl Phys Lett 1996; 68: 2793-2795.

20 Hädrich S, Klenke A, Hoffmann A, Eidam T, Gottschall T et al. Nonlinear compression to sub-30-fs, $0.5 \mathrm{~mJ}$ pulses at $135 \mathrm{~W}$ of average power. Opt Lett 2013; 38: 3866-3869.

21 Hädrich S, Klenke A, Rothhardt J, Krebs M, Hoffmann A et al. High photon flux tabletop coherent extreme ultraviolet source. Nat Phot 2014; 8: 779-783.

22 Rothhardt J, Hädrich S, Klenke A, Demmler S, Hoffmann A et al. 53 W average power few-cycle fiber laser system generating soft $\mathrm{x}$ rays up to the water window. Opt Lett 2014; 39: 5224-5227.

23 Ozawa A, Rauschenberger J, Gohle C, Herrmann M. High harmonic frequency combs for high resolution spectroscopy. Phys Rev Lett 2008; 100: 253901.

24 Pupeza I, Holzberger S, Eidam T, Carstens H, Esser D et al. Compact high-repetitionrate source of coherent $100 \mathrm{eV}$ radiation. Nat Phot 2013; 7: 608-612.

25 Allison TK, Cingöz A, Yost DC, Ye J. Extreme nonlinear optics in a femtosecond enhancement cavity. Phys Rev Lett 2011; 107: 183903.

26 Kim S, Jin J, Kim Y-J, Park I-Y, Kim Y et al. High-harmonic generation by resonant plasmon field enhancement. Nature 2008; 453: 757-760.

27 Sivis M, Duwe M, Abel B, Ropers C. Extreme-ultraviolet light generation in plasmonic nanostructures. Nat Phys 2013; 9: 304-309.

28 Vernaleken A, Weitenberg J, Sartorius T, Russbueldt P, Schneider W et al. Single-pass high-harmonic generation at $20.8 \mathrm{MHz}$ repetition rate. Opt Lett $2011 ; 36$ : 3428-3430.

29 Blättermann A, Chiang C, Widdra W. Atomic line emission and high-order harmonic generation in argon driven by $4-\mathrm{MHz}$ sub- $\mu \mathrm{J}$ laser pulses. Phys Rev A 2014; 89: 043404.

30 Rundquist A, Durfee CG, Chang Z, Herne C, Backus S et al. Phase-matched generation of coherent soft X-rays. Science 1998; 280: 1412-1415.

31 Pupeza I, Högner M, Weitenberg J, Holzberger S, Esser D et al. Cavity-enhanced highharmonic generation with spatially tailored driving fields. Phys Rev Lett 2014; 112 . 103902.

32 Klenke A, Breitkopf S, Kienel M, Gottschall T, Eidam T et al. 530 W, $1.3 \mathrm{~mJ}$ four-channel coherently combined femtosecond fiber chirped-pulse amplification system. Opt Lett 2013; 38: 2283-2285.

33 Active Fiber Systems. Available at http://www.afs-jena.de (accessed 30 September 2014).

34 Amplitude Systémes. Available at http://www.amplitude-systemes.com (accessed 30 September 2014).

35 Saraceno CJ, Emaury F, Schriber C, Hoffmann M, Golling M et al. Ultrafast thin-disk laser with $80 \mu \mathrm{J}$ pulse energy and $242 \mathrm{~W}$ of average power. Opt Lett 2014; 39: 9-12.

36 Brons J, Pervak V, Fedulova E, Bauer D, Sutter D et al. Energy scaling of Kerr-lens mode-locked thin-disk oscillators. Opt Lett 2014; 39: 6442-6445.

37 Travers JC, Chang W, Nold J, Joly NY, St J Russell P. Ultrafast nonlinear optics in gas-filled hollow-core photonic crystal fibers [Invited]. J Opt Soc Am B 2011; 28: A11-A26.

38 Emaury F, Dutin CF, Saraceno CJ, Trant M, Heckl OH et al. Beam delivery and pulse compression to sub-50 fs of a modelocked thin-disk laser in a gas-filled Kagome-type HC-PCF fiber. Opt Express 2013; 21: 4986-4994.

39 Mak KF, Travers JC, Joly NY, Abdolvand A, St J Russell P. Two techniques for temporal pulse compression in gas-filled hollow-core kagomé photonic crystal fiber. Opt Lett 2013; 38: 3592-3595. 
40 Emaury F, Saraceno CJ, Debord B, Ghosh D, Diebold A et al. Efficient spectral broadening in the $100-W$ average power regime using gas-filled kagome HC-PCF and pulse compression. Opt Lett 2014; 39: 6843-6846.

41 Möller M, Cheng Y, Khan SD, Zhao B, Zhao K et al. Dependence of high-orderharmonic-generation yield on driving-laser ellipticity. Phys Rev A2012; 86: 011401.

42 Salières $P$, L'Huillier A, Lewenstein $M$. Coherence control of high-order harmonics. Phys Rev Lett 1995; 74: 3776-3779.

43 Constant E, Garzella D, Breger P, Mevel E, Dorrer C et al. Optimizing high harmonic generation in absorbing gases: model and experiment. Phys Rev Lett 1999; 82 : 1668-1671.

44 Kazamias S, Daboussi S, Guilbaud O, Cassou K, Ros D et al. Pressure-induced phase matching in high-order harmonic generation. Phys Rev A 2011; 83: 063405

45 Ammosov MV, Delone NB, Krainov VP. Tunnel ionisation of complex atoms and of atomic ions in an altering electromagnetic field. Sov Phys JETP 1986; 64 : 1191-1194.

46 Scoles G. Atomic and Molecular Beam Methods. Oxford University Press; 1988.
47 Henke BL, Gullikson EM, Davis JC. X-ray interactions: photoabsorption, scattering, transmission, and reflection at $\mathrm{E}=50-30000 \mathrm{eV}, \mathrm{Z}=1-92$. At Data Nucl Data Tables 1993; 54: 181-342.

48 Haarlammert T, Zacharias $\mathrm{H}$. Application of high harmonic radiation in surface science. Mater Sci 2009; 13: 13-27.

49 Sandberg RL, Paul A, Raymondson DA, Hädrich S, Gaudiosi DM et al. Lensless diffractive imaging using tabletop coherent high-harmonic soft-X-ray beams. Phys Rev Lett 2007; 99: 098103.

cc) (i) $\odot$ This license allows readers to copy, distribute and transmit the Contribution as long as it attributed back to the author. Readers may not alter, transform or build upon the Contribution, or use the article for commercial purposes. Please read the full license for further details at - http://creativecommons.org/licenses/ by-nc-nd/4.0/

Supplementary Information for this article can be found on the Light: Science \& Applications' website (http://www.nature.com/lsa/). 